\title{
Open-source software in medical imaging: development of OsiriX
}

\author{
Osman Ratib • Antoine Rosset
}

Received: 9 January 2006 / Accepted: 27 September 2006 / Published online: 15 November 2006

(C) CARS 2006

\begin{abstract}
Purpose Open source software (oss) development for medical imaging enables collaboration of individuals and groups to produce high-quality tools that meet user needs. This process is reviewed and illustrated with OsiriX, a fast DICOM viewer program for the Apple Macintosh.

Materials and methods OsiriX is an oss for the Apple Macintosh under Mac OS X v10.4 or higher specifically designed for navigation and visualization of multimodality and multidimensional images: 2D Viewer, 3D Viewer, 4D Viewer (3D series with temporal dimension, for example: Cardiac-CT) and 5D Viewer (3D series with temporal and functional dimensions, for example: Cardiac-PET-CT). The 3D Viewer offers all modern rendering modes: multiplanar reconstruction, surface rendering, volume rendering and maximum Intensity projection. All these modes support 4D data and are able to produce image fusion between two different series (for example: PET-CT). OsiriX was developed using the Apple Xcode development environment and Cocoa framework as both a DICOM PACS workstation for medical imaging and an image processing software package for medical research (radiology and nuclear imaging), functional imaging, 3D imaging, confocal microscopy and molecular imaging.

Results OsiriX is an open source program by Antoine Rosset, a radiologist and software developer, was designed specifically for the needs of advanced imaging modalities. The software program turns an Apple
\end{abstract}

O. Ratib $(\varangle) \cdot$ A. Rosset

Department of Radiology and Medical Informatics,

University Hospital of Geneva, 24, rue Micheli-du-Crest,

1205 Geneva, Switzerland

e-mail: osman.ratib@hcuge.ch
Macintosh into a DICOM PACS workstation for medical imaging and image processing. OsiriX is distributed free of charge under the GNU General Public License and its source code is available to anyone. This system illustrates how open software development for medical imaging tools can be successfully designed, implemented and disseminated.

Conclusion oss development can provide useful cost effective tools tailored to specific needs and clinical tasks. The integrity and quality assurance of open software developed by a community of users does not follow the traditional conformance and certification required for commercial medical software programs. However, open software can lead to innovative solutions designed by users better suited for specific tasks.

\section{Introduction}

Medical imaging techniques are becoming a major component of patient management and clinical decision making in a trend towards evidence-based medicine that rely heavily on objective findings for diagnostic and therapeutic tasks. Consequently, the need for image visualization and analysis has extended beyond the traditional diagnostic arena in radiology departments and is becoming common practice in all medical subspecialty. Most clinical decisions rely on imaging procedure where the diagnostic report generated by a radiologist is often incomplete without supporting images. It is also not uncommon that radiologists have to generate additional images using volume rendering and image processing techniques to better communicate their findings to clinicians and surgeons. 
Furthermore, three-dimensional (3D) imaging has rapidly become an essential part of the visualization tools used in a variety of diagnostic procedures in radiology. With the current generation of ultrafast CTs or MRIs it is possible to acquire very large high-resolution volumetric data in few seconds. These studies provide isotropic data that have identical resolution in three dimensions providing exquisite detailed anatomical information. With 3D volume rendering techniques it is possible to generate high-quality images offering a realistic anatomical view of the body and organs from tomographic data [1]. These images offer an attractive alternative to traditional display of axial images, allowing users to get a better perception of the topology and special relationship of different structures in the body. However, 3D rendering and multiplanar data formatting will only be useful in clinical routine if they are easily accessible and fast enough to be generated and manipulated in real time.

The recent technical innovations in image modalities allow to acquire temporal sequences fast enough to observe and analyze dynamic behaviors and physiological mechanisms. With the latest generations of CT and MRI scanners we can now acquire sequences of images showing the transit of contrast agents throughout the body or through a given organ [2]. With this kind of improved capability for acquiring fast images, it is also possible to acquire dynamic sequences of rapidly moving anatomical structures such as a beating heart for example [3]. These temporal images are often referred to as a fourth dimension of the imaging data.

Another fast growing domain of medical imaging is the evolution toward functional and molecular imaging. These modalities are able to produce images that represent an in vivo characterization of biological processes at the cellular and molecular level. Positron emission tomography (PET) using radio-labeled tracers represents the most common molecular imaging modality [4], but other techniques based on molecular markers are nowadays emerging in other modalities such as MRI offering new perspectives of functional imaging and a great hope to increase the specificity and sensibility of diagnostic and detection of varieties of diseases [5]. Theses modalities are complementary to classic conventional and anatomical imaging such as CT and MRI. The ability to combine the functional or molecular data with the anatomical images adds a new dimension to the images. This functional or molecular component is often referred to as the fifth dimension [6].

Visualization and processing of $3 \mathrm{D}, 4 \mathrm{D}$ and $5 \mathrm{D}$ data require special navigation tools and multidimensional rendering software that are often only available on high- end 3D rendering workstations that are only accessible in academic and specialized imaging centers [7]. Ironically, where these tools are needed the most is in clinical services and in areas where clinical decisions and patient management choices are being made. They are also becoming a communication means for radiologists to communicate their findings. The availability of these tools are, however, very limited outside radiology departments due to their high cost and complex setups.

Development of commercial systems driven by medical imaging manufacturers lags behind the increasing demand for advanced processing tools. They usually follow research and clinical validation and require several years before releasing new products on the market. These are the reasons why open source and free software is becoming more widely adopted in the medical community. Open source not only provides a cost effective alternative, but because they are being developed by a community of developers from the field, the software tools can be customized to match the needs and specific usage in clinical setups outside radiology. This new strategy in software development was adopted in our project for the development of a new image visualization and processing platform called OsiriX. The goal of this project was to develop a software platform for multidimensional and multi-modality image visualization and image processing. By adopting the open source paradigm we rapidly benefited from the support and help of the scientific and medical community around the world. Hundreds of institutions adopted the program and helped identifying its limitation and flaws and contributed to its improvement and its robustness by finding different bugs and flaws. Furthermore, numerous developers have embarked in the project and contributed to the development of the software code.

\section{Inadequacy of market-driven engineering}

In a recent editorial by David Channin in Radiology [8], he elegantly demonstrated that the traditional market-driven engineering is mostly inadequate for coping for today's needs in medical imaging and medical informatics in general. In this traditional model the major vendors try to guess what the next feature of their product should be on the basis of feedback from clinical users of their devices and leaders in the field. They then try to validate this guess work on the basis of their experience with existing products in the field. Unfortunately in this traditional model the features that we the customers of the imaging equipment tend to focus on are the specific processing tools that we need for the particular imaging modality without enough insight in the informatics 
and image-driven clinical communication tasks that are needed on a broader clinical scope. Furthermore it is difficult to adequately predict the tools that are needed for better image navigation and visualization if the users can only rely on the existing models. As David Channin puts it "...the most difficult thing to see in an image is something that is not there, and the most difficult thing to foresee in an information system is a feature that is not present...".

The major difficulty in the conventional marketdriven model adopted by vendors is that there is limited room for innovative and experimental explorations of new avenues with a reasonable turnaround time that could allow users to rapidly evaluate new features. Also the input from advanced users is usually limited and "filtered" by the manufacturers' engineering and marketing teams that will only allow changes in areas with limited financial risk and minimal deviation from the main architecture of the existing products. This is the reason why widely adopted open source platforms could become a very attractive alternative solution for manufacturers as well, allowing them to explore and develop new tools involving the users themselves before incorporating these new tools into their line of products. In this new paradigm, manufacturers can contribute to and benefit from the open source community with limited risk and much faster identification of innovative solutions that can have a real impact on day-to-day usage of their products in clinical practice. In an extended model similar to the well-known "Red Hat" model of the Linux operating system, it is possible to envision new business models based on the integration and support of open source components into commercial settings. New commercial avenues will then include service providers that will ensure proper integration, certification and quality insurance, as well as maintenance and user training and support for open source products that are developed by a large community of users that cannot provide these services.

\section{What is open source?}

The concept of open source software (OSS) promotes the development and sharing of software source code under special licensing agreements that protects author's copyrights while maintaining the distribution of free and open derivative work based on the original code. The most successful example of open source development is Linux Operating system. Numerous other oss products have been widely adopted such Apache web server software that has by far exceeded the number of implementation sites of competing commercial products. While the most successful and well-known example of open source is the development of Linux by Linus Torvalds in 1991, other charismatic defenders of this new strategy of software development such as Richard Stallman played a major role in the growth and success of open source paradigm. Richard Stallman founded the GNU (GNU is a recursive acronym for "GNU's Not UNIX") project in 1984 and the Free Software Foundation in 1985. He also contributed significantly to the widespread adoption of open source by authoring a number of well known and highly used development tools, including the GNU Compiler Collection (GCC), the GNU symbolic debugger (GDB) and GNU Emacs.

In medicine, open source has gained momentum only in the recent years. Early adopters have mainly focused on applications that were mostly applicable in research areas. One of the early adopter and promoter of free and open source application in medical imaging is Wayne Rasband at NIH, the author of NIH Image http://rsb. info.nih.gov/nih-image/ and later of ImageJ http://rsb. info.nih.gov/ij/ two software programs dedicated to image manipulation and image processing. Similarly in the early 1990s Osman Ratib and his team at the University Hospital of Geneva developed OSIRIS, a multiplatform DICOM viewer intended for clinical users [12].

The rationale behind open source is very simple: when programmers can read, redistribute and modify the source code for a piece of software, the software evolves. People improve it, adapt it, and fix bugs. And this can happen at a speed that, if one is used to the slow pace of conventional software development, seems astonishing. The rapidly growing open source community has realized that this rapid evolutionary process produces better software than the traditional closed model, in which only a very few programmers can see the source code and everybody else must blindly use an opaque set of software tools.

It is important to acknowledge that not all the free software programs are open source. Numerous software packages are distributed as "shareware" by which the software is provided free of charge or in exchange for a voluntary token contribution, but the source code is kept as a property of its main author. While shareware software benefit from broad distribution and feedback from users they lack the ability of improvement from input and enhancement provided by other developers and users.

Several non-profit organizations have emerged to support and help the development and the success of open source initiatives. open source Initiative (OSI) is a non-profit corporation dedicated to managing and promoting the open source definition for the good of the community, specifically through the OSI certified 
open source software certification mark and program (http://www.opensource.org/). In healthcare, several smaller organizations have emerged such as the open source in health Care (OSHCA) association (http://www.oshca.org/), and open source in healthcare (http://www.openhealth.org/), and more specifically for medical imaging the OpenRad initiative (http://www. openrad.com/). In a broader spectrum, on 15 August 2006 OSDL hosted the first ever Healthcare Day at LinuxWorld Expo. The proceedings are available for "Opening Up Healthcare - How Open Source Software, Standards and Solutions are Changing the Healthcare Industry" at http://osdl.org/wiki/dcl_health_care _day/index.php/Main_Page. This is certainly not a comprehensive list and there are numerous small associations and users groups that are active in the domain of oss development in medicine. Of interest are online "Journal of Free and Open Source Medical Computing" http://www.josmc.org/ and "LINUX Medical News" (http://www.linuxmednews.com/) where news and information about Open Source developments in medicine can be found.

Numerous open source imaging and PACS software package are already available and slowly gaining momentum. The main characteristic of the open software is that it is being peer reviewed and evaluated by the user community, and only the best will gather enough momentum and be widely adopted. To be successful, an open source project must have a strong development momentum with a leading team or preferably a single leader that ensures the consistency and quality of the software that is being distributed. Given that most open source project cannot be funded by traditional channels such as grants or industry support, most of the work is provided by benevolent contributors and the most challenging task always relies on the main project manager team that will rapidly be overwhelmed by the users' demands and also by the growing community of developers. Most successful projects were always driven by benevolent dictator(s) that directed the software development and maintained high quality and high productivity of software release and a constant pace. Without such leadership and long-term commitment, most projects will rapidly fade out and fail the transition from a small local development to a broad international project. Most projects will also rapidly encounter significant financial difficulties and shortage of resources to pursue the growth of project developments. Given the limited number of funding sources only academic groups that have sufficient sources of research funds and development resources can carry out large open source projects over long periods of time A list of recent open source initiatives in medical imaging software can be found at http://www.idoimaging.com/. In the scientific community, a new set of open source libraries have emerged for the visualization of multidimensional data. The most successful set of tools is provided by Insight Software Consortium (http://www.insightsoftwareconsortium. org/): The Visualization Toolkit or VTK (http://www. public.kitware.com/VTK/) is a well recognized and widely adopted software library that runs on multiple platforms and has been used for numerous scientific and medical applications so far. The recent adjunction of the Insight Toolkit or ITK (http://www.itk.org/), mostly funded by the US National Library of Medicine as part of the Visible Human Project, adds a wealth of additional rendering and image processing tools for medical applications. More information on VTK and ITK applications can be found in the "Insight Journal" (http://www.insight-journal.org/). There is also an emerging effort to consolidate multiple projects into larger community of non-profit associations that can have a stronger core momentum for fund raising and sharing of development resources. Among such initiatives one should mention a consortium of academic groups that recently launched a concerted effort for the development of open source components for PACS and image management in medicine and holding its first general conference on "Open Source Strategy for Multi-Center Image Management" (http://www.mcim. georgetown.edu) in March 2006. The first objective of this new consortium is to regroup its partners' efforts in open source development under a common framework called image management tool kit (IMTK).

\section{Impact of open-source in medicine}

Numerous recent reports showed that the evolution and adoption of electronic medical records (EMR) in medicine have significantly lagged behind expectations due to three major barriers: excessive cost, the transience of vendors and the lack of common data standards [9]. Many authors suggested, however, that oss reduces barriers to EMR adoption, first by reducing EMR ownership and development costs. Open source offers freedom from software licensing costs with reduced cost software upgrades and no license expiration. The medical informatics community welcomes OSS, which fits naturally its scientific model of shared, peer-reviewed knowledge in medicine. Vendors of open source applications can share development costs with the user community and focus on implementation and support services. The resulting paradigm shift is that open source medical system vendors can become professional service providers, 
competing on service quality rather than on the basis of software secrets.

The impact of open source is even greater in specialized areas of medicine such as medical imaging. These vertical markets have always been a challenge for vendors and manufacturers due to the small size of specialized users and high expectations in terms of complexity and performance of the tools that users need. This has naturally driven the market to high-end and high-cost developments and marketing strategies that also try to cope with very rapid evolution of computer technologies and software developments that make most products obsolete in very short time interval, which does not allow the manufacturers to generate sustainable return on investment. In very specialized niches such as in medical imaging it is almost impossible to maintain profitable business models that are self-sustained. Most manufacturers will cover the cost through revenues from other business avenues such as sales of imaging modalities, scanners and imaging devices or by providing expensive global solutions and charging high costs for implementation and support and maintenance contracts of complex integrated information systems.

\section{Quality assurance and compliance with regulations}

The major criticism often expressed against oss products is that it is quite difficult to maintain an official quality assurance program and it is also often not possible to comply with local and governmental regulations and requirements. In most cases, oss components are of higher quality and robustness than commercial counterparts because they are being developed and debugged by a larger community of users and software developers that continuously improve the integrity and performance of the software code. It is, however, difficult for the community of developers of open source products to carry the burden of officially certifying the software products and complying with local governmental laws and regulations applicable to software programs used in clinical practice. In the open source paradigm these features will be provided by separate commercial entities and service providers that can provide and support certified versions of the software tools for a service fee. Similar to the "Red Hat" commercial version of Linux operating system, it is anticipated that "White Hat" commercially supported versions of open source medical applications could be provided specifically for the medical market in compliance with local regulations.

In the United States, the Food and Drug Administration (FDA) imposes certain rules and regulations to medical software components in the context of medi- cal devices. This requires manufacturers to file specific applications for pre-market submissions for software contained in medical devices. While such certification does not require extensive validation efforts it does carry a certain cost and legal responsibility that most open source developers would not be willing to support given that the software is being distributed free of charge. There is, however, a trend in academic community and in some large scientific organizations as well as in some governmental agencies to start a new thought process regarding regulations and quality insurance of medical software. The trend is to aim for new guidelines and regulations based on the concept of GMP (good manufacturing process) requirements applied in other medical domains and in particular in drug manufacturing. The purpose being to ensure the adequacy of quality control mechanisms and software validation processes more than the specific certification of a given software tool. Other major efforts are being made to ensure consistency and compatibility between different software component and applications. The major effort in medical imaging is being handled under the IHE initiative that provides guidelines and mechanisms for adequate management of data and workflow for interoperability between different systems and software programs [10]. For an oss to be successful and widely adopted it will need to comply with IHE workflow schema and medical imaging standards to be able to integrate into existing commercial systems.

\section{OsiriX: example of open source imaging platform}

In a collaboration effort, the University of California in Los Angeles (UCLA) and the University Hospital of Geneva (HUG) elected to develop a multi-modality image viewing and navigation software called OsiriX on Apple Macintosh computers [11]. The goal of our project is to develop a completely new software platform that will allow users to efficiently and conveniently navigate through large sets of multidimensional data without the need of high-end expensive hardware or software. We also elected to develop our system on new oss libraries allowing the system to be easily adaptable to a variety of hardware platform. We also benefit from our past experience of developing one of the first free DICOM viewers several years ago called OSIRIS running on Macintosh and Windows personal computers [12] and distributed as a shareware to the user community and the source code made available to academic institutions. The success and wide adoption of the OSIRIS software has encouraged us to extend our effort into 
a completely new program that would be more suitable for navigating through large multidimensional data sets.

In our design we also wanted to take advantage of the significant progress in performance and flexibility of 3D animation on personal computers mostly driven by the computer graphics and game industry [13]. Most video games today are developed on OpenGL graphic libraries that benefit from hardware acceleration and processing capabilities of today's ultra-fast video cards. Also, OpenGL being an industry standard, it will automatically adapt to any hardware configuration and take advantage of any hardware accelerator that is provided for video display of $2 \mathrm{D}$ and $3 \mathrm{D}$ data. In the scientific community, a new set of open source libraries have emerged for the visualization of multidimensional data. The most successful set of tools provided by Insight Software Consortium are The Visualization Toolkit or VTK (http://www.public.kitware.com/VTK/) and the Insight Toolkit or ITK (http://www.itk.org/), providing additional rendering and image processing tools for medical applications. These new open source software toolkits offer powerful functions to do complex images manipulations, and great performance for real-time 3D images visualization. Our objective was to incorporate these powerful tools in a new graphic user interface (GUI) that is more suitable for clinical applications and image interpretation of large multidimensional datasets. We made special efforts to design a software platform that allows to easily and quickly develop new generation of multidimensional viewers that could replace many of the existing functions available only on high-end expensive $3 \mathrm{D}$ workstations.

In the OsiriX open source project we also we elected to explore, evaluate and integrate new emerging technologies to expand capabilities of PACS beyond its current limitations [14]. We intentionally selected general consumer products for their wide availability, low cost, convenience and ease of use. We selected a set of new products and services recently released by Apple Computer (http://www.apple.com) and adapted them for medical imaging applications: (1) the iPod, a popular portable music player, was integrated to serve as a highcapacity portable DICOM storage with a high-speed transfer rate. (2) iChat AV instant messaging and videoconferencing software was adapted to allow real-time radiology videoconferencing tool for remote image viewing and screen sharing. (3) iDisk, an Internet service provided by Apple for secure data storage on a virtual hard disk was adopted as a DICOM data storage and communication of DICOM images.

The software includes features that allow users to instantly send an email with the image (or image sets) that are being reviewed on the screen. One can also ini-

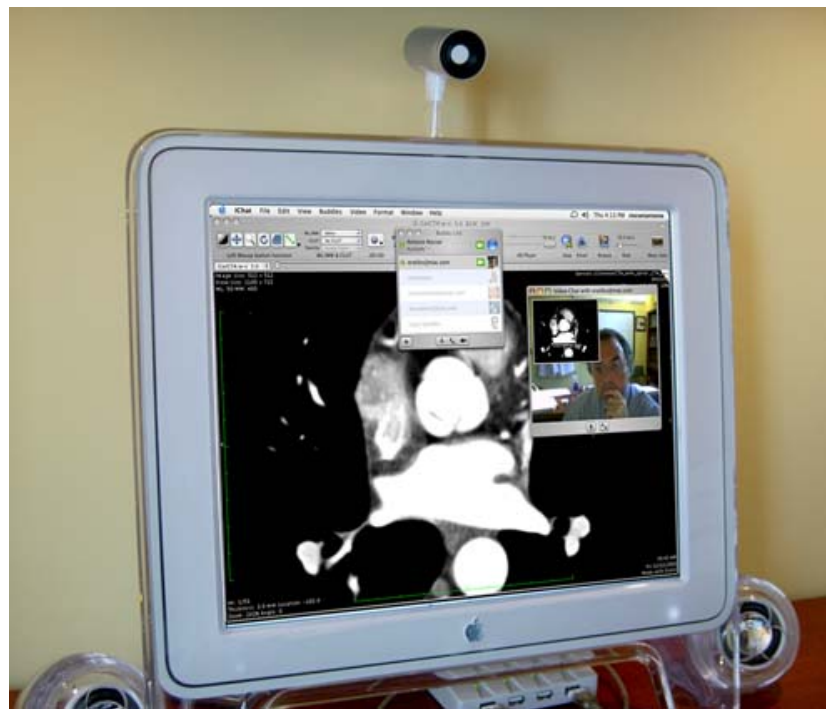

Fig. 1 Illustration of a teleconferencing setup using iChat instant messaging service provided on the Apple platform. This feature is accessible directly from within the OsiriX program allowing users to share the content of a displayed image using a simple teleradiology feature

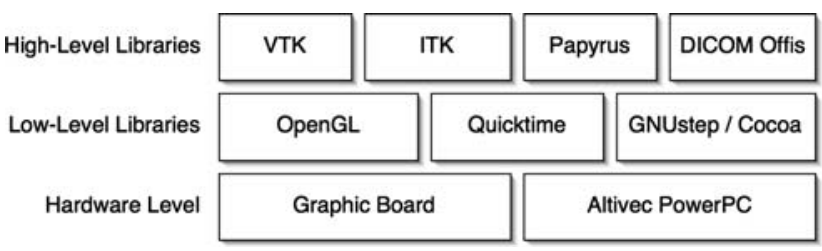

Fig. 2 General software architecture of OsiriX software based on open source components

tiate a live conference with any other OsiriX user on the network (or on the internet) using the iChat application that was adapted to allow the user to replace the live video image by a live view of the content of the window that is displayed on the screen (see Fig. 1). This allows interactively showing and discussing any image in real time providing a simple and convenient mechanism for teleradiology and remote consultations.

\section{Software architecture}

The OsiriX program was developed on the new Unixbased apple Macintosh platform under Os X version 10.4 (see Fig. 2). For the design and development of our software platform we selected the following standard components and tools:

(1) "Openstep/GNUStep/Cocoa/": an object-oriented framework for the development of the graphical user interface. This framework is an object-oriented environment that offers graphic programming tools and allows to quickly design and modify user interfaces in a graph- 
Fig. 3 Examples of advanced image visualization and processing tools for multi-dimensional and multimodality imaging showing different display and visualization tools applicable for four-dimensional dynamic cardiac CT images

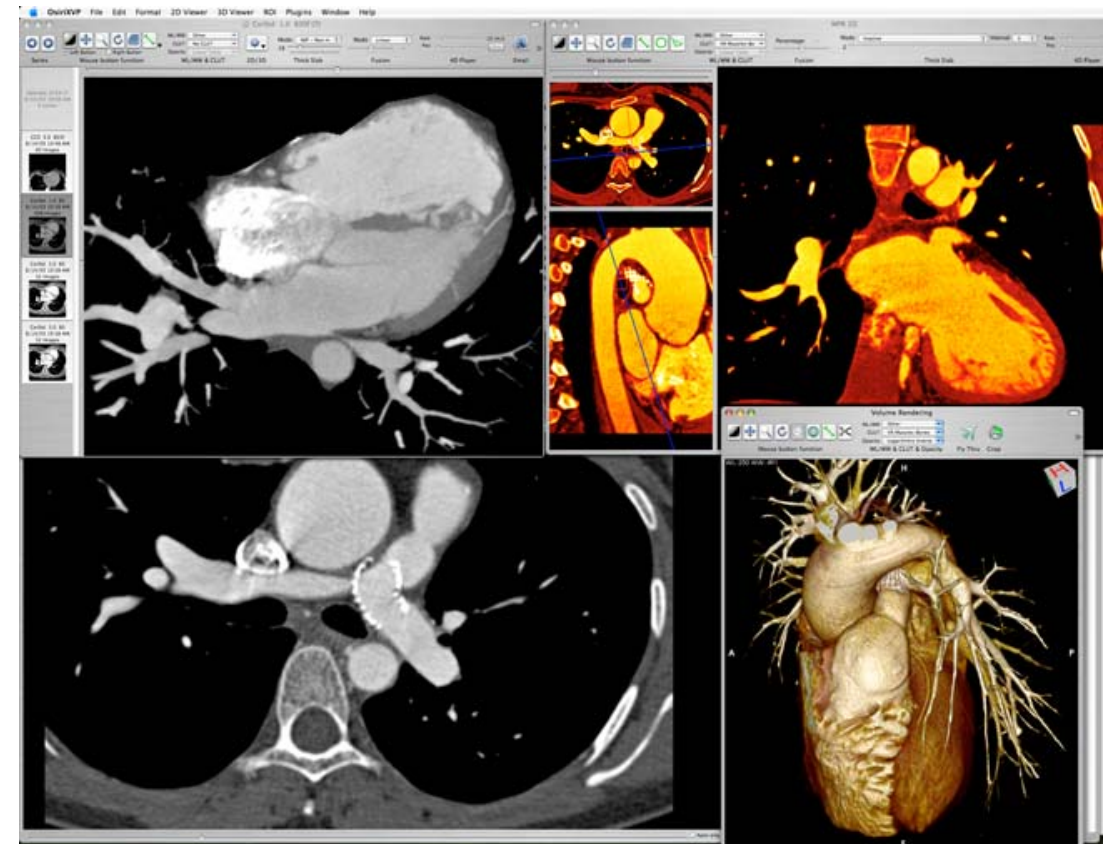

ical environment. The first version of this framework was created on the Next platform: NextStep. It has then evolved as an open source project under the OpenStep/GNUStep [15] name and was chosen by Apple Computer as the developing framework under the Cocoa name for their MacOS X operating system.

(2) OpenGL: an industry standard graphic library for the 2D and 3D image visualization functions. This standard library allows to take advantage of hardware acceleration by $3 \mathrm{D}$ graphic cards. It allows to manipulate large datasets directly on the graphic card, avoiding multiple transfers between the computer memory (RAM) and graphic card memory.

(3) The Visualization Toolkit (VTK): an extensive library open source software tool for 3D image processing and visualization that is widely adopted in the scientific community. This toolkit offers a large number of functions to manipulate and display 3D data and is based on OpenGL for ensuring the best performance on different hardware platforms. The VTK toolkit is a $\mathrm{C}++$ framework that supports all standard 3D reconstructions: surface rendering, multi-planar reconstruction (MPR), maximum intensity projection and volume rendering [16].

(4) The Insight Segmentation and Registration Toolkit (ITK) is an extended set of software tool for specific image processing, segmentation and registration functions. This toolkit is an extension to the VTK toolkit and is based on the same framework philosophy. It is also available as a $\mathrm{C}++$ framework [17].

(5) "Papyrus toolkit" for DICOM files management: This public domain library offers all needed functions to read and write DICOM files including all meta-data. It is an ANSI C library [18].

(6) "DICOM Offis" for DICOM network functions: This library offers network functions to query, send, retrieve and receive DICOM images in a PACS network. This toolkit is a C++ library [19].

(7) PixelMed for DICOM network functions: PixelMed is a Java toolkit that supports the entire DICOM syntax for transfers and file manipulation [20].

The most important aspect of the project is that all the components function together within a simple and userfriendly GUI. To achieve the best possible user interface design, we chose to develop the OsiriX program on a Macintosh platform to benefit from its well-known user interface features and convenience and ease of use (see Fig. 3). Additionally, the latest development environment provided by Apple greatly facilitates the rapid development of interactive graphic applications.

In a recent software update we also adopted an advanced peer-to-peer communication mechanism based on the Open Bonjour protocol that is imbedded in the Apple operating system. This mechanism allows multiple users on the network to share the content of their local image database as well as easy access to multiple DICOM image servers accessible over the network (see Fig. 4).

Our clinical testing after integration of PSIRIX workstations with our existing PACS network showed significant better performance of the OsiriX platform compared to conventional PACS workstation in image retrieval and image display and manipulation. Even 3D rendering and reformatting tools compared favorably 


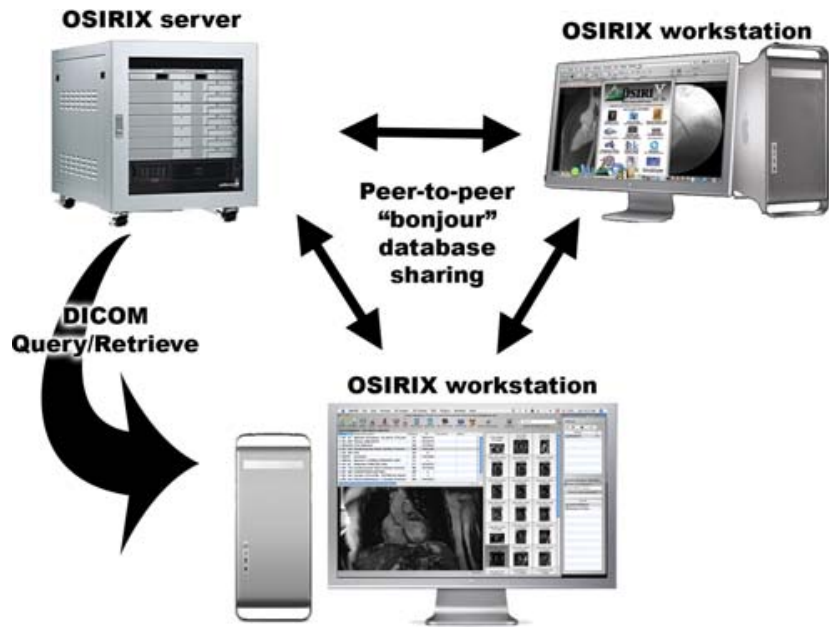

Fig. 4 Diagram illustrating a peer-to-peer architecture using bonjour technology for remote consultation of image data between different workstations on the network and facilitated access to multiple sources of DICOM image data from multiple servers

with traditional 3D processing workstations. While it was difficult to conduct controlled benchmark comparison of workstation performance, it is important to outline two of the technical features that support the performance of the OsiriX platform on Apple Macintosh hardware:

- Multithreading: OsiriX is specially written to exploit the latest technologies in multiprocessing computing with multiprocessors and multicores systems. A large number of the software tools use all the processors/cores available for complex processing tasks such as 3D rendering, compression/decompression, resampling, rescaling, etc.)

- SIMD (Single Instruction - Multiple Data) Units: Recent generation processors such as Intel and PowerPC processors offer special computing units (Altivec for PowerPC and SSE3 for Intel) that are able to process large chunk of data (128 or 256 bit) in a single processor instruction. These units allow an acceleration of up to 8 times faster, compared to "classic" processors instructions. These SIMD units are particularly useful in digital signal processing for imaging because post-processing functions require to apply the same algorithm to large amount of data. In OsiriX, all resampling, rescaling, units conversions use SIMD optimized algorithms.

The OsiriX program as well as the source code can be downloaded from http://www.homepage.mac.com/ rossetantoine/osirix/. An online discussion group is accessible at http://www.tech.groups.yahoo.com/group/ osirix/ with over 1,500 members exchanging over 300 messages every month. Documentation and user manual posted by users and developers can be found on the Wiki web site http://www.en.wikibooks.org/wiki/Online_ Osirix_Documentation.

\section{Discussion and conclusion}

Open source software is an idea whose time has finally come. For 20 years it has been building momentum in the technical cultures that built the Internet and the World Wide Web. Now it is breaking out into the commercial world and into vertical markets such as medical applications, and that is changing all the rules. It is particularly promising in advanced image display and analysis applications where the rapid increase in demand cannot be matched by traditional expensive commercial solutions. The evolution of radiology imaging modalities from $2 \mathrm{D}$ sectional acquisition to a $3 \mathrm{D}$ volumetric acquisition and even higher dimensions with functional data and the emergence of multimodality scanners such as PET/CT scanners calls for new and innovative display and visualization tools. To allow radiologists and clinicians to conveniently and efficiently interpret these large exams, traditional image viewers have to be re-designed and adapted to a new paradigm of multidimensional image navigation, visualization and manipulation. By combining multiple new technologies and open source projects it is today possible to develop a new generation of high-performance 3D DICOM viewers for personal computers. These 3D visualization tools were traditionally only accessible on expensive 3D workstations restricting their use to specialist radiologists. With the incorporation of these multidimensional navigation and visualization tools in standard and easily accessible image viewing program they should rapidly be adopted as a standard way of image interpretation in clinical settings for patient management and clinical decision making.

However, with the introduction of more advanced image processing and visualization functions, imaging software tend to become more complex and usually include numerous processing functions that are not necessarily useful for all the users. Depending on the type of images being evaluated and the interpretation task to be performed, most users would only use a fraction of the tools or features of a typical image processing and $3 \mathrm{D}$ visualization program. The major advantage of open source developments is the ability to rapidly explore new techniques and allow easy customization of software programs for different users and applications. Each user should be able to customize the software to include 
only the functions and tools that are needed for their specific needs. In short open source developments offer the opportunity to innovate extravagantly and then let the users' winnow selections for suitable and useful tools and features that they need.

In our project of development and wide distribution of OsiriX we experienced the tremendous potential of open source paradigm inside a community of specialists and professional users that were extremely supportive and instrumental in making the success of this initiative go beyond our wildest expectations. The rapid growth of OsiriX features and tools was driven by the large community of users that contributed to its constant improvement in response to real practical needs. It is a perfect example of software application development directly driven by users and tailored for their specific needs. The most challenging task for our development team was to keep the pace and coordinate the development of new tools while maintaining the robustness and stability of the whole program. Thanks to the wealth of feedback and input from thousands of users around the world it was possible to release software updates and new features at a rate that exceeded by far the rate of software updates in the industry. This was even more challenging on the Macintosh platform due to the extremely rapid evolution of the computer operating system and hardware platforms that were updated by Apple at a rate of major enhancements of the operating system every 6 months and a change in the architecture and performance of the hardware platforms twice or three times a year.

Besides the development of new processing and analysis tools that will continue, it is our plan is to extend the OsiriX platform to become more than just a visualization and image processing software. A prototype of a server version of OsiriX using the core database and communication features of OsiriX to manage a large capacity storage volume provides a simple solution for building the main component of a min-PACS that can serve a network of imaging devices and processing workstations. By supporting all standard DICOM communication features, it provides a cost effective solution when used with new rapid storage technology like the ones released by Apple computer allowing to set up over 7 Terabytes of storage capacity for less than $\$ 10,000$. In our institution we implemented such a solution to provide an extension to our commercial PACS for storage of very large datasets generated by multidetector scanners generating extremely large number of thin image slices used for generating 3D image and volume rendering of anatomical structures. Usually these large data sets (of over hundreds of gigabytes) cannot be stored on the clinical PACS due to their size and the impact on image communication that they generate on a conventional PACS. Such an addition to existing PACS can provide an elegant solution for temporary storage of those large volumes of images with impacting the performance and workflow of existing PACS.

Furthermore we joined a larger consortium of open source developer in medical imaging to contribute to the development of a larger range of software tools that will support image and data management in clinical and multicentric research projects. The first kick-off meeting of this new initiative was held during a 2-day workshop in Las Vegas in March 2006 called "Open Source Strategy for Multi-Center Image Management" (http://www.mcim.georgetown.edu). The consortium is currently extending its efforts in open source development under a common framework called Image Management Tool Kit (IMTK). A second workshop is planned for May 2007.

\section{References}

1. Salgado R, Mulkens T, Bellinck P et al (2003) Volume rendering in clinical practice, a pictorial review. JBR-BTR 86:215220

2. Flohr T, Ohnesorge B, Bruder H et al (2003) Image reconstruction and performance evaluation for ECG-gated spiral scanning with a 16-slice CT system. Med Phys 30:2650-2662

3. Saito K, Saito M, Komatu S et al (2003) Real-time four-dimensional imaging of the heart with multi-detector row CT. Radiographics 23:E8-8

4. Lemke AJ, Niehues SM, Hosten N, Amthauer H, Boehmig M, Stroszczynski C, Rohlfing T, Rosewicz S, Felix R (2004) Retrospective digital image fusion of multidetector CT and 18F-FDG PET: clinical value in pancreatic lesions-a prospective study with 104 patients. J Nucl Med 45(8):1279-1286.

5. Spadola L, Rosset A, Seeger L, Motamedi K, Ratib O (2005) Color Fusion MRI: an effective technique for image visualization in a variety of clinical applications. In: SPIE proceedings, Medical Imaging 2005, PACS and Imaging Informatics vol 5748, p 463

6. Ratib O, Rosset A, Dahlbom M, Czernin J (2005) Navigating the fifth dimension: new concepts in interactive multimodality and multidimensional image navigation. In: SPIE proceedings, Medical Imaging 2005, PACS and Imaging Informatics 2005, vol 5748, p 28

7. Ratib O (2004) PET/CT image navigation and communication. J Nucl Med 45 (Suppl 1):46S-55S

8. Channin D.S. (2003) Driving market-driven engineering. (Editorial). Radiology 229:311-313

9. Kantor GS, Wilson WD, Midgley A (2003) Open-source software and primary care EMR. J Am Informet Assoc 10:616

10. Channin D.S (2002) Integrating the healthcare enterprise: a primer, The fellowship of IHE. Radiographics 22:1555-1560

11. Rosset A, Spadola L, Ratib O (2004) OsiriX: an open source software for navigating in multidimensional DICOM images. J Digital Imaging 17(3):205-216

12. Ratib O, Ligier Y, Mascarini C, Logean M, Girard C, Trayser G, Hochstrasser D (1997) Multimedia image and data navigation workstation. Radiographics 17(2):515-521 
13. Rosset A, Spadola L, Ratib O (2004) OsiriX: a new generation of multidimensional DICOM viewer based on new imaging standards. Comput Assisted Radiol Surgery (CARS 2004 Proc) 1268:1247

14. Chantal R., Antoine R., Osman, R (2005) General consumer communication tools for improved image management and communication in medicine. J. Digital Imaging 18(4): 270-279

15. GNU GCC Compiler. http://www.gcc.gnu.org, accessed September 2006

16. The Visualization Toolkit (VTK): http://www.public. kitware.com/VTK/, accessed September 2006
17. The Insight Segmentation and Registration Toolkit (ITK): http://www.itk.org/, accessed September 2006

18. Papyrus Toolkit, Digital Imaging Unit, Geneva University Hospital: http://www.sim.hcuge.ch/papyrus/01_Papyrus _Presentation_EN.htm, accessed September 2006

19. DICOM Offis Toolkit: http://www.dicom.offis.de, accessed September 2006

20. PixelMed Java Toolkit: http://www.pixelmed.com/, accessed September 2006 